\title{
Plumbagin inhibits cell proliferation and promotes apoptosis in multiple myeloma cells through inhibition of the PI3K/Akt-mTOR pathway
}

\author{
HONGWEI WU ${ }^{1 *}$, XIAOZHEN DAI ${ }^{2 *}$ and ENREN WANG ${ }^{3}$ \\ ${ }^{1}$ Department of Hematology, The First Affiliated Hospital of Chengdu Medical College; \\ ${ }^{2}$ Department of Biological and Medical Sciences, Chengdu Medical College; ${ }^{3}$ Department of Neuromedicine, \\ The First Affiliated Hospital of Chengdu Medical College, Chengdu, Sichuan 610500, P.R. China
}

Received March 27, 2015; Accepted April 22, 2016

DOI: $10.3892 / \mathrm{ol} .2016 .5048$

\begin{abstract}
Plumbagin is the primary component of the traditional Chinese medicine Baihua Dan, and possesses anti-infection and anticancer effects with the ability to enhance the sensitivity of tumor cells to radiation therapy. The present study aimed to investigate the potential anticancer effect and mechanism of plumbagin on multiple myeloma (MM) cells. Human MM OPM1 cells were treated with plumbagin, and its impact on cell viability, cytotoxicity, apoptosis and caspase-3 activity was examined by 3-(4,5-dimethylthiazol-2-yl)-2,5-diphenyltetrazolium bromide, lactate dehydrogenase leakage, flow cytometry and colorimetric assays. In addition, the protein expression levels of phosphoinositide 3-kinase, phosphorylated (p)-Akt and p-mammalian target of rapamycin (mTOR) in OPM1 cells were analyzed by western blotting. The results demonstrated that plumbagin treatment inhibited cell viability, increased cell cytotoxicity, activated cell apoptosis and promoted caspase- 3 activity in the OPM1 cells. Furthermore, pretreatment of plumbagin significantly suppressed PI3K, p-Akt and p-mTOR protein expression levels in the OPM1 cells. In conclusion, the present study indicates that plumbagin inhibits cell proliferation and promotes apoptosis in MM cells through inhibition of PI3K/Akt-mTOR expression.
\end{abstract}

\section{Introduction}

Multiple myeloma (MM) is a type of malignant tumor that originates from B cell lines (1). The disease is characterized by an increase in the number of abnormal plasma cells, which generate monoclonal immunoglobulin, and a malignant

Correspondence to: Mr. Enren Wang, Department of Neuromedicine, The First Affiliated Hospital of Chengdu Medical College, 278 Baoguang Avenue, Chengdu, Sichuan 610500, P.R. China E-mail: enrenwang1234567@163.com

*Contributed equally

Key words: plumbagin, multiple myeloma, PI3K/Akt-mTOR proliferation within bone marrow, causing fracture and bone marrow function failure, which results in the clinical symptoms of MM, including bone pain, anemia, hypercalcemia, infection and renal failure (1). Without treatment, patients with progressive stages of MM have a median survival of only 6 months, whilst following chemotherapy, the median survival is $>3$ years $(2,3)$. Only $25 \%$ of patients with MM survive for $>5$ years; therefore, MM has long been regarded as an incurable disease with an urgent requirement for novel therapies to improve the prognosis of patients $(2,3)$.

Phosphoinositide 3-kinase (PI3K)/Akt/mammalian target of rapamycin (mTOR) is an important signaling pathway that affects the energy metabolism, size, cycle, proliferation, survival and apoptosis of cells, and is closely associated with numerous other signaling pathways $(4,5)$. The administration of drugs that target the PI3K-AKT-mTOR signaling pathway, combined with additional therapy, is a promising approach for patients with MM (2).

Plumbagin, a natural naphthoquinone compound, is the primary active component of the traditional Chinese medicine Baihua Dan (6). Baihua Dan has been administered clinically in China for centuries, and the effects of plumbagin include anti-inflammatory (7), antiseptic (8) and anti-protozoa (9) effects. Plumbagin functions through a variety of pathways to inhibit and kill tumor cells; it was previously demonstrated that plumbagin induces apoptosis in human lung cancer A549 cells by inhibiting the tissue plasminogen activator (PA)-induced expression of matrix metalloproteinases and urokinase PA (10). Subsequently the metastasis of tumor cells is reduced. However, the anticancer effect of plumbagin on $\mathrm{MM}$ and the precise molecular mechanisms underlying its behavior remain unclear. Therefore, the present study aimed to investigate the effects and molecular mechanisms of plumbagin on the proliferation and apoptosis of MM cells.

\section{Materials and methods}

Chemicals and reagents. RPMI-1640, fetal bovine serum (FBS), 3-(4,5-dimethylthiazol-2-yl)-2,5-diphenyltetrazolium bromide (MTT) and lactate dehydrogenase (LDH) were all purchased from Sigma-Aldrich (St. Louis, MO, USA). The 
chemical structure of plumbagin (purity, 98\%; Sigma-Aldrich) is presented in Fig. 1. A Pierce ${ }^{\mathrm{TM}}$ BCA Protein Assay kit was purchased from Thermo Fisher Scientific, Inc. (Waltham, MA, USA) and the Caspase-3 Colorimetric Assay kit was purchased from Beyotime Institute of Biotechnology, Inc. (Nanjing, China).

Cell lines and cell culture. Human MM OPM1 cells were provided by the Experiment Center of The First Affiliated Hospital of Chengdu Medical College (Chengdu, China). OPM1 cells were cultured in RPMI-1640 containing $10 \% \mathrm{FBS}$ and $1 \%$ penicillin and streptomycin at $37^{\circ} \mathrm{C}$ in a humidified atmosphere with $5 \% \mathrm{CO}_{2}$.

MTT assay. OPM1 cells were seeded at a density of 8,000 cells/well into a 96 -well culture plate. After $24 \mathrm{~h}$, the OPM1 cells were treated with plumbagin at doses of 0 (dimethyl sulfoxide vehicle-only), 1, 5, 10, 20 and $50 \mu \mathrm{M}$ (11), for 24 and $48 \mathrm{~h}$. Following treatment, $10 \mu \mathrm{l}$ MTT stock solution $(5 \mathrm{mg} / \mathrm{ml})$ was added to each well and incubated for $4 \mathrm{~h}$ at $37^{\circ} \mathrm{C}$ in a humidified atmosphere with $5 \% \mathrm{CO}_{2}$. A total of $150 \mu \mathrm{l}$ DMSO was added to each well to dissolve the crystals, and cell viability was subsequently measured at a wavelength of $450 \mathrm{~nm}$.

LDH leakage. OPM1 cells were seeded at a density of 8,000 cells/well into a 96-well culture plate. After $24 \mathrm{~h}$, the OPM1 cells were treated with plumbagin $(0,10$, 20 and $50 \mu \mathrm{M}$ ) for 24 and $48 \mathrm{~h}$. Following treatment, $100 \mu \mathrm{l}$ LDH solution was added to each well and incubated for $30 \mathrm{~min}$ at $37^{\circ} \mathrm{C}$ in a humidified atmosphere with $5 \% \mathrm{CO}_{2}$. Absorbance was measured using an enzyme-linked immunosorbent assay reader at $490 \mathrm{~nm}$.

Cellular apoptosis analysis by flow cytometry. OPM1 cells were seeded at a density of $10^{6}$ cells/well into a 6 -well culture plate. After $24 \mathrm{~h}$, the OPM1 cells were treated with plumbagin $(0,10,20$ and $50 \mu \mathrm{M})$ for $24 \mathrm{~h}$. Following treatment, the cells were washed and fixed in precooled PBS, resuspended with buffer solution and incubated at room temperature with Annexin V-fluorescein isothiocyanate for $30 \mathrm{~min}$ in darkness. Subsequently, the cells were incubated with propidium iodide for $30 \mathrm{~min}$ in darkness. Cell apoptosis was enumerated using a Coulter ${ }^{\circledR}$ Epics $\mathrm{XL}^{\mathrm{TM}}$ flow cytometer (Beckman Coulter, Inc., Brea, CA, USA).

Caspase-3 activity. OPM1 cells were seeded at a density of $10^{6}$ cells/well into a 6 -well culture plate. After $24 \mathrm{~h}$, the cells were treated with plumbagin $(0,10,20$ and $50 \mu \mathrm{M})$ for $24 \mathrm{~h}$. Following treatment, the caspase- 3 activity in fluorescence was detected at a wavelength of $405 \mathrm{~nm}$ using a Caspase-3 Colorimetric Assay kit (Beyotime Institute of Biotechnology), according to the manufacturer's protocol.

Western blot analysis. OPM1 cells were seeded into a 6-well culture plate at a density of $10^{6}$ cells/well. After $24 \mathrm{~h}$, the cells were treated with plumbagin $(0,10,20$ and $50 \mu \mathrm{M})$ for $24 \mathrm{~h}$. Following treatment with plumbagin, the cells were harvested and lysed in radioimmunoprecipitation assay buffer. Total protein was determined using a Pierce ${ }^{\mathrm{TM}}$ BCA Protein assay

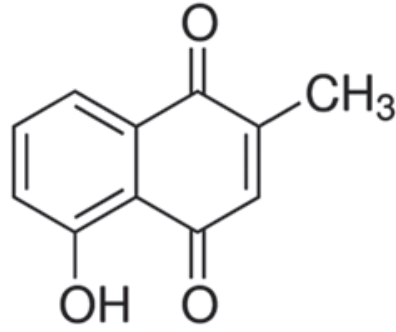

Figure 1. Chemical structure of plumbagin.

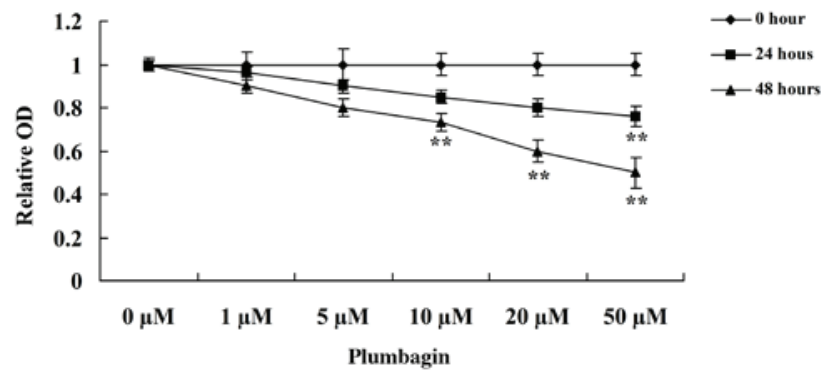

Figure 2. Plumbagin inhibits the proliferation of human multiple myeloma OPM1 cells. Data are presented as the mean \pm standard deviation. ${ }^{* *} \mathrm{P}<0.01$ vs. $0 \mu \mathrm{M}$ plumbagin.

kit. Equal amounts of protein were loaded and separated by 7-12\% sodium dodecyl sulfate-polyacrylamide gel electrophoresis and subsequently transferred to a polyvinylidene fluoride membrane. The membrane was then blocked with $5 \%$ skimmed milk and probed with the following primary antibodies: Anti-PI3K (\#sc-48637; dilution, 1:1,000), anti-phosphorylated (p)-Akt1 (\#sc-135650; dilution, 1:2,000), anti-p-mTOR (\#sc-101738; dilution, 1:1,000) and anti- $\beta$-actin (\#sc-130656; dilution, 1:1,000) antibodies (Santa Cruz Biotechnology, Inc.) overnight at $4^{\circ} \mathrm{C}$. The membrane was washed with Tris-buffered saline with Tween-20, incubated with anti-rabbit secondary antibody (\#6401-05; dilution, 1:5,000; Amyjet Scientific, Inc., Wuhan, China) at $4^{\circ} \mathrm{C}$ for $1 \mathrm{~h}$, and visualized in an enhanced chemiluminescence solution (GE Healthcare Life Sciences, Little Chalfont, UK). Protein expression was then detected in a ChemiDoc MP Imaging System (Bio-Rad Laboratories, Inc., Hercules, CA, USA).

Statistical analysis. Data are presented as the mean \pm standard deviation of three independent experiments and statistical analysis was performed using SPSS software version 17.0 (SPSS, Inc., Chicago, IL, USA). Statistical differences between the control and treatment samples were determined by one-way analysis of variance. $\mathrm{P}<0.05$ was considered to indicate statistically significant differences.

\section{Results}

Plumbagin inhibits cell proliferation. The present study assessed the anticancer effect of plumbagin on OPM1 cell viability using MTT assay. Incubation of the OPM1 cells with $0,1,5,10,20$ and $50 \mu \mathrm{M}$ plumbagin resulted in significantly reduced cell viability (Fig. 2). Following treatment with 10, 


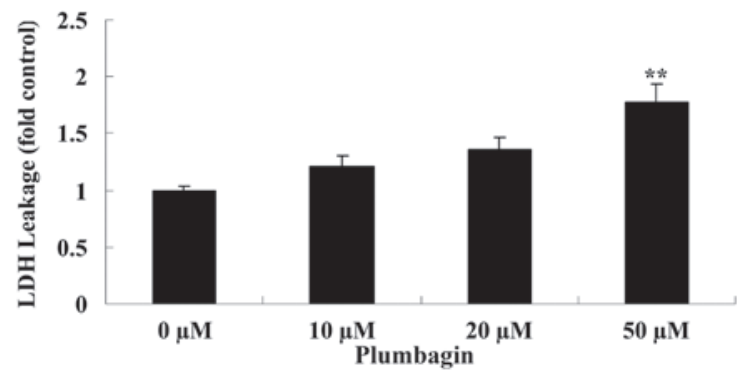

Figure 3. Plumbagin increased the cytotoxicity of human multiple myeloma OPM1 cells. Data are presented as the mean \pm standard deviation. ${ }^{* *} \mathrm{P}<0.01$ vs. $0 \mu \mathrm{M}$ plumbagin. LDH, lactate dehydrogenase.

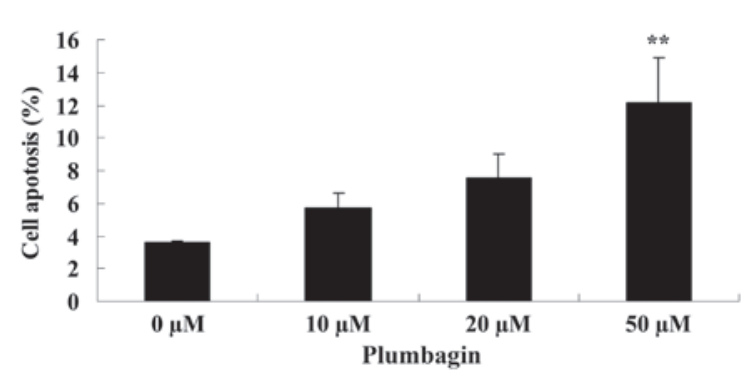

Figure 4. Plumbagin induces cell apoptosis of human multiple myeloma OPM1 cells. Data are presented as the mean \pm standard deviation. ${ }^{* *} \mathrm{P}<0.01$ vs. $0 \mu \mathrm{M}$ plumbagin.

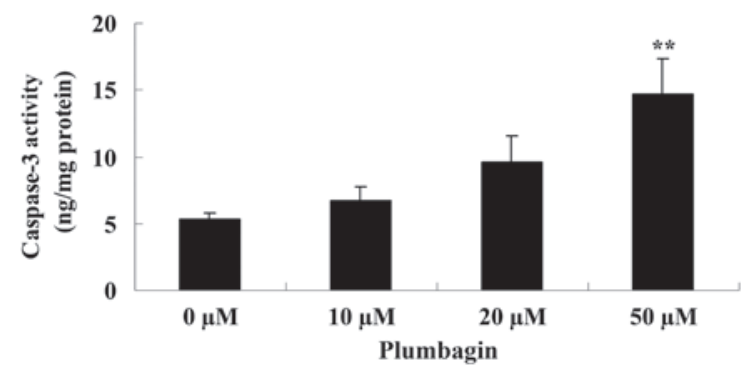

Figure 5. Plumbagin increases caspase-3 activity in human multiple myeloma OPM1 cells. Data are presented as the mean \pm standard deviation. . ${ }^{* *} \mathrm{P}<0.01$ vs. $0 \mu \mathrm{M}$ plumbagin.

20 and $50 \mu \mathrm{M}$ plumbagin for $48 \mathrm{~h}$, and $50 \mu \mathrm{M}$ plumbagin for $24 \mathrm{~h}$, the viability of the OPM1 cells significantly decreased $(\mathrm{P}=0.0003)$. These results indicate that plumbagin has a potent anticancer effect on the proliferation of OPM1 cells.

Plumbagin increases cell cytotoxicity. The current study subsequently examined the anticancer effect of plumbagin on OPM1 cell cytotoxicity by assessing LDH leakage. Treatment of the OPM1 cells with $50 \mu \mathrm{M}$ plumbagin resulted in significantly increased cell cytotoxicity at $24 \mathrm{~h}$ compared with cells treated with $0 \mu \mathrm{M}$ plumbagin $(\mathrm{P}<0.0001$; Fig. 3$)$.

Plumbagin induces cell apoptosis. Next, the anticancer effect of plumbagin on OPM1 cell apoptosis was analyzed by flow cytometry. Following treatment with $50 \mu \mathrm{M}$ plumbagin for $24 \mathrm{~h}$, it was observed that OPM1 cell apoptosis significantly increased compared with cells treated with $0 \mu \mathrm{M}$ plumbagin ( $\mathrm{P}=0.0018$; Fig. 4).
A

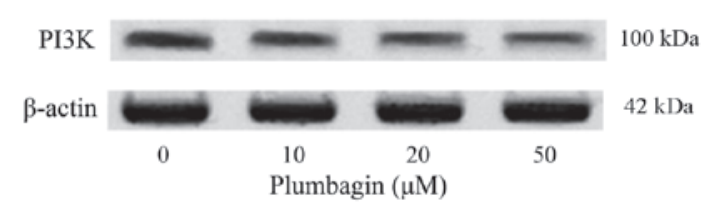

B

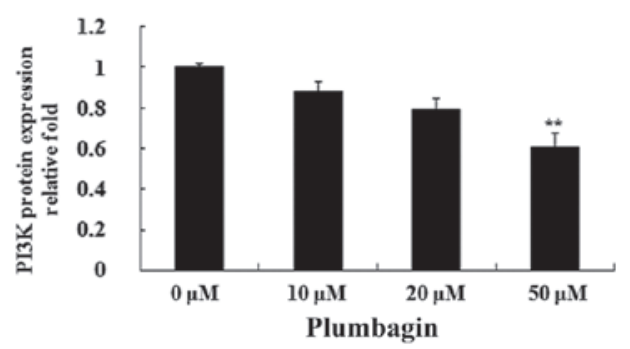

C

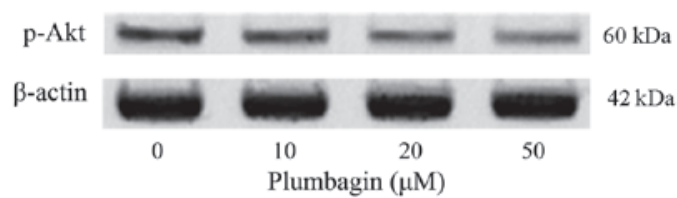

D

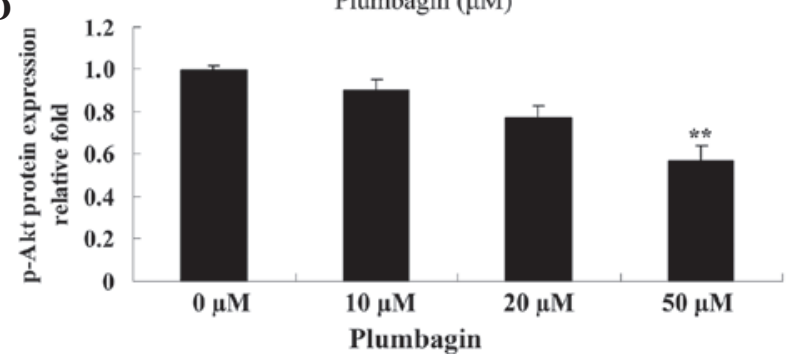

Figure 6. Plumbagin inhibits PI3K/Akt protein expression in human multiple myeloma OPM1 cells. Western blotting demonstrated that plumbagin inhibited (A and B) PI3K and (C and D) p-Akt protein expression. Data are presented as the mean \pm standard deviation. ${ }^{* *} \mathrm{P}<0.01$ vs. $0 \mu \mathrm{M}$ plumbagin. PI3K, phosphoinositide 3-kinase; p-, phosphorylated.
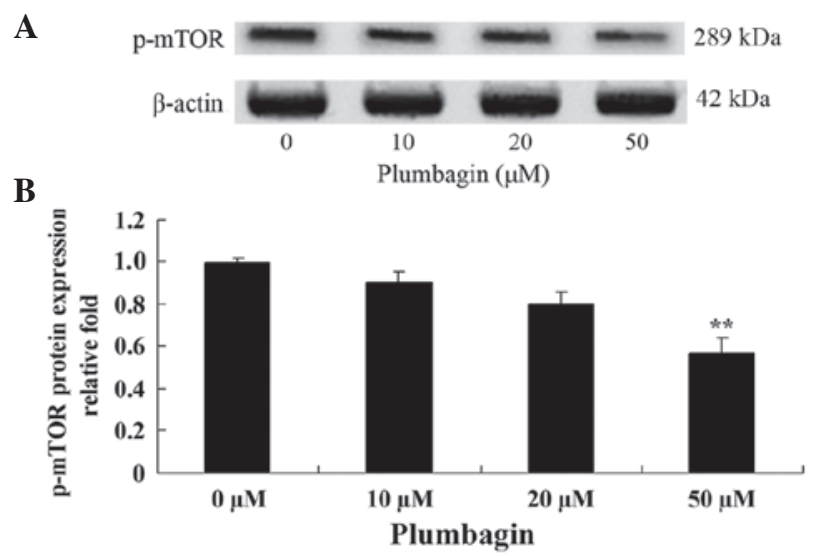

Figure 7. Plumbagin inhibits mTOR in human multiple myeloma OPM1 cells. (A and B) Western blotting demonstrated that plumbagin inhibited p-mTOR protein expression. Data are presented as the mean \pm standard deviation. ${ }^{* *} \mathrm{P}<0.01$ vs. $0 \mu \mathrm{M}$ plumbagin. mTOR, mammalian target of rapamycin; $\mathrm{p}-$, phosphorylated.

Plumbagin induces caspase-3 activity. The present study examined the anticancer effect of plumbagin on caspase-3 activity in OPM1 cells following a $24 \mathrm{~h}$ treatment time. The results demonstrated that incubation with $50 \mu \mathrm{M}$ plumbagin for $24 \mathrm{~h}$ significantly increased caspase- 3 activity in the OPM1 cells compared with cells treated with $0 \mu \mathrm{M}$ plumbagin $(\mathrm{P}<0.0001$; Fig. 5). 
Plumbagin inhibits PI3K/Akt. The effect of plumbagin on the protein expression levels of PI3K and p-Akt was evaluated using western blot analysis, which revealed that the anticancer effect of plumbagin is via the PI3K/Akt signaling pathway in OPM1 cells. Following incubation with $50 \mu \mathrm{M}$ plumbagin for $24 \mathrm{~h}$, the OPM1 cells exhibited significantly decreased expressions of PI3K and p-Akt ( $\mathrm{P}<0.0001$; Fig. 6).

Plumbagin inhibits mTOR. The present study investigated the anticancer effect of plumbagin on the mTOR signaling pathway in OPM1 cells, which were treated with the plumbagin for $24 \mathrm{~h}$. The results demonstrated that treatment of the OPM1 cells with $5 \mu \mathrm{M}$ plumbagin significantly reduced the expression level of $\mathrm{p}-\mathrm{mTOR}(\mathrm{P}=0.0003$ Fig. 7).

\section{Discussion}

$\mathrm{MM}$ is a common malignant plasma cell proliferative disease of the bone marrow, which accounts for $10-15 \%$ of all malignant blood diseases (12). Even though life expectancy and diagnostics are improving for this disease, its incidence is increasing, and fractures and clinical complications, including, renal failure, are also on the rise $(13,14)$. The results of the present study demonstrated that treatment with plumbagin significantly reduced cell viability, increased cell cytotoxicity, activated cell apoptosis and promoted caspase-3 activity in OPM1 cells. In addition, previous studies have reported that plumbagin suppresses the growth of oral squamous cell carcinoma (15), colon cancer (16) and breast cancer (17) cells.

It has been previously demonstrated that the overexpression and abnormal activation of PI3K and Akt is involved in the occurrence and development of a number of tumors, including ovarian, breast, pancreatic, stomach and non-small cell lung cancer (3). Akt, a type of serine/threonine protein kinase, is essential in PI3K signal transmission. The pleckstrin homology domain of Akt binds to the activation products of PI3K, and Akt becomes phosphorylated and activated. Subsequently, active Akt regulates downstream target genes, which are involved in cell proliferation, differentiation and apoptosis (18). In the present study, pretreatment with plumbagin significantly suppressed the expression of PI3K and p-Akt in OPM1 cells. Similarly, Li et al (19) reported that plumbagin induced apoptosis in human non-small cell lung cancer cells through inhibition of the PI3K/Akt/mTOR pathway. Furthermore, Wang et al (11) observed that plumbagin induced cell cycle arrest in human pancreatic cancer cells via the PI3K/Akt/mTOR-mediated pathway.

The downstream effects of PI3K and Akt are often identified in patients with cancer (3). mTOR is an important downstream molecule of Akt, which is essential in tumorigenesis (20). In the PI3K/Akt-mTOR signaling pathway, Akt negatively regulates two tumor suppressor genes: PTEN, which is upstream of Akt, and Tuberous Sclerosis Complex 1 and 2, which are located downstream of AKT and upstream of mTOR (21-23). Downstream effects, conserved throughout protein evolution, of forkhead box O (FOXO) transcription factors and mTOR are important in tumorigenesis (22). In mammalian cells, Akt is able to phosphorylate a number of proteins (24); FOXO transcription factors may be directly phosphorylated and inactivated by Akt, and under normal physiological conditions,
FOXO suppresses mammalian cell proliferation (25). By contrast, mTOR, which promotes cell proliferation, is indirectly activated by Akt (25). The results of the current study indicated that treatment with plumbagin significantly reduced the expression level of p-mTOR in OPM1 cells. Similarly, Chen et al (26) reported that plumbagin induced cell apoptosis and inhibited cell growth in human colon cancer cells via the PI3K/Akt-mTOR signaling pathway.

In conclusion, the results of the present study demonstrate that plumbagin inhibits cell proliferation and promotes apoptosis of MM cells. In addition, the present study identified a potential cellular mechanism of plumbagin in MM cells, which was the PI3K/Akt/mTOR signaling pathway. Additional studies are required to confirm the therapeutic effect of plumbagin for its use in clinical trials in patients with MM.

\section{References}

1. Liu N, Zhou H, Yang G, Geng C, Jian Y, Guo H and Chen W: Retrospective analysis of genetic abnormalities and survival in 131 patients with multiple myeloma. Oncol Lett 9: 930-936, 2015.

2. Tan E, Weiss BM, Mena E, Korde N, Choyke PL and Landgren O: Current and future imaging modalities for multiple myeloma and its precursor states. Leuk Lymphoma 52: 1630-1640, 2011.

3. Wang F, Zhang W, Guo L, Bao W, Jin N, Liu R, Liu P, Wang Y, Guo Q and Chen B: Gambogic acid suppresses hypoxia-induced hypoxia-inducible factor- $1 \alpha /$ vascular endothelial growth factor expression via inhibiting phosphatidylinositol 3-kinase/Akt/mammalian target protein of rapamycin pathway in multiple myeloma cells. Cancer Sci 105: 1063-1070, 2014

4. Deng M, Wang J, Chen Y, Zhang L and Liu D: Combination of SF1126 and gefitinib induces apoptosis of triple-negative breast cancer cells through the PI3K/AKT-mTOR pathway. Anticancer Drugs 26: 422-427, 2015.

5. Zhang H, Bajraszewski N, Wu E, Wang H, Moseman AP, Dabora SL, Griffin JD and Kwiatkowski DJ: PDGFRs are critical for PI3K/Akt activation and negatively regulated by mTOR. J Clin Invest 117: 730-738, 2007.

6. Padhye S, Dandawate P, Yusufi M, Ahmad A and Sarkar FH: Perspectives on medicinal properties of plumbagin and its analogs. Med Res Rev 32: 1131-1158, 2012.

7. Wang T, Wu F, Jin Z, Zhai Z, Wang Y, Tu B, Yan W and Tang T: Plumbagin inhibits LPS-induced inflammation through the inactivation of the nuclear factor-kappa B and mitogen activated protein kinase signaling pathways in RAW 264.7 cells. Food Chem Toxicol 64: 177-183, 2014.

8. Inbaraj JJ and Chignell CF: Cytotoxic action of juglone and plumbagin: A mechanistic study using $\mathrm{HaCaT}$ keratinocytes. Chem Res Toxicol 17: 55-62, 2004.

9. Saowakon N, Lorsuwannarat N, Changklungmoa N, Wanichanon C and Sobhon P: Paramphistomum cervi: The in vitro effect of plumbagin on motility, survival and tegument structure. Exp Parasitol 133: 179-186, 2013.

10. Shieh JM, Chiang TA, Chang WT, Chao CH, Lee YC, Huang GY, Shih YX and Shih YW: Plumbagin inhibits TPA-induced MMP-2 and u-PA expressions by reducing binding activities of NF-kappaB and AP-1 via ERK signaling pathway in A549 human lung cancer cells. Mol Cell Biochem 335: 181-193, 2010.

11. Wang F, Wang Q, Zhou ZW, Yu SN, Pan ST, He ZX, Zhang X, Wang D, Yang YX, Yang T, et al: Plumbagin induces cell cycle arrest and autophagy and suppresses epithelial to mesenchymal transition involving PI3K/Akt/mTOR-mediated pathway in human pancreatic cancer cells. Drug Des Devel Ther 9: 537-560, 2015.

12. Troeltzsch M, Oduncu F, Mayr D, Ehrenfeld M, Pautke C and Otto S: Root resorption caused by jaw infiltration of multiple myeloma: Report of a case and literature review. J Endod 40: 1260-1264, 2014

13. Que W, Li S and Chen J: NS-398 enhances the efficacy of bortezomib against RPMI8226 human multiple myeloma cells. Mol Med Rep 7: 1641-1645, 2013.

14. Nakazato T, Sagawa M and Kizaki M: Triptolide induces apoptotic cell death of multiple myeloma cells via transcriptional repression of Mcl-1. Int J Oncol 44: 1131-1138, 2014. 
15. Ono T, Ota A, Ito K, Nakaoka T, Karnan S, Konishi H, Furuhashi A, Hayashi T, Yamada Y, Hosokawa Y and Kazaoka Y: Plumbagin suppresses tumor cell growth in oral squamous cell carcinoma cell lines. Oral Dis 21: 501-511, 2015.

16. Eldhose B, Gunawan M, Rahman M, Latha MS and Notario V: Plumbagin reduces human colon cancer cell survival by inducing cell cycle arrest and mitochondria-mediated apoptosis. Int J Oncol 45: 1913-1920, 2014.

17. Dandawate P, Ahmad A, Deshpande J, Swamy KV, Khan EM, Khetmalas M, Padhye S and Sarkar F: Anticancer phytochemical analogs 37: Synthesis, characterization, molecular docking and cytotoxicity of novel plumbagin hydrazones against breast cancer cells. Bioorg Med Chem Lett 24: 2900-2904, 2014.

18. Fuchs O: Targeting of NF-kappaB signaling pathway, other signaling pathways and epigenetics in therapy of multiple myeloma. Cardiovasc Hematol Disord Drug Targets 13: 16-34, 2013.

19. Li YC, He SM, He ZX, Li M, Yang Y, Pang JX, Zhang X, Chow K, Zhou Q, Duan W, et al: Plumbagin induces apoptotic and autophagic cell death through inhibition of the PI3K/Akt/mTOR pathway in human non-small cell lung cancer cells. Cancer Lett 344: 239-259, 2014.

20. Koldehoff M, Beelen DW and Elmaagacli AH: Inhibition of mTOR with everolimus and silencing by vascular endothelial cell growth factor-specific siRNA induces synergistic antitumor activity in multiple myeloma cells. Cancer Gene Ther 21: 275-282, 2014.

21. Gay F, Oliva S, Petrucci MT, Conticello C, Catalano L, Corradini P, Siniscalchi A, Magarotto V, Pour L, Carella A, et al: Chemotherapy plus lenalidomide versus autologous transplantation, followed by lenalidomide plus prednisone versus lenalidomide maintenance, in patients with multiple myeloma: a randomised, multicentre, phase 3 trial. Lancet Oncol 16: 1617-1629, 2015.
22. Yang Y, Zhou X, Xiao M, Hong Z, Gong Q, Jiang L and Zhou J: Discovery of chrysoeriol, a PI3K-AKT-mTOR pathway inhibitor with potent antitumor activity against human multiple myeloma cells in vitro. J Huazhong Univ Sci Technolog Med Sci 30: 734-740, 2010.

23. Dong M, Yang G, Liu H, Liu X, Lin S, Sun D and Wang Y: Aged black garlic extract inhibits HT29 colon cancer cell growth via the PI3K/Akt signaling pathway. Biomed Rep 2: 250-254, 2014.

24. Chen X, Yang C, Xu Y, Zhou H, Liu H and Qian W: The microtubule depolymerizing agent CYT997 effectively kills acute myeloid leukemia cells via activation of caspases and inhibition of PI3K/Akt/mTOR pathway proteins. Exp Ther Med 6: 299-304, 2013.

25. Jang J, Jeong SJ, Kwon HY, Jung JH, Sohn EJ, Lee HJ, Kim JH, Kim SH, Kim JH and Kim SH: Decursin and doxorubicin are in synergy for the induction of apoptosis via STAT3 and/or mTOR pathways in human multiple myeloma cells. Evid Based Complement Alternat Med 2013: 506324, 2013.

26. Chen MB, Zhang Y, Wei MX, Shen W, Wu XY, Yao C and Lu PH: Activation of AMP-activated protein kinase (AMPK) mediates plumbagin-induced apoptosis and growth inhibition in cultured human colon cancer cells. Cell Signal 25: 1993-2002, 2013. 